\title{
Phenylalanine intercalation parameters for liquid-disordered phase domains - a membrane model study
}

\author{
Paulina Adamczewski and Valeria Tsoukanova * (D)
}

\begin{abstract}
Background: Propensity of phenylalanine (Phe) for nonpolar environments drives its intercalation into phospholipid membranes, which has been implicated in metabolic and neurological disorders. The knowledge of Phe intercalation parameters can be instrumental in understanding various membrane processes triggered by interactions with Phe, in particular the early events leading to the formation of nucleation/docking sites for the selfassembly of Phe amyloid fibrils at the membrane surface.

Results: In this study, we used monolayers of phosphatidylethanolamine (DPPE) and phosphatidylcholine (DPPC) to mimic the membrane outer leaflet. Its initial interaction with Phe was modeled by injecting Phe into the aqueous phase underneath the monolayer. Constant pressure insertion assays augmented with epifluorescence microscopy imaging were used to monitor Phe intercalation. Our primary goal was to determine the Phe intercalation area, $A_{\text {phe. }}$ Two values were obtained for $A_{\text {phe, }} 33 \pm 2$ and $48 \pm 3 \AA^{2}$.

Conclusions: Phe appeared to discriminate between DPPE and DPPC packing, and use two modes of intercalation. The area of $A_{\text {Phe }} 33 \pm 2 \AA^{2}$ is consistent with a Phe monomer intercalating into membrane by inserting the phenyl ring nearly normal to the membrane surface. This mode has been found to operate in DPPE membranes. For DPPC membranes however, the value of $A_{\text {phe }}=48 \pm 3 \AA^{2}$ suggests that, from saline, Phe tends to intercalate as a larger species plausibly dragging along a counterion, $\mathrm{Na}^{+}$, in a $\mathrm{Na}^{+}$(Phe) complex.
\end{abstract}

Keywords: Phenylalanine, Langmuir monolayer, Epifluorescence microscopy, Amino acid/phospholipid interaction, Amyloid fibrils

\section{Background}

Phenylalanine (Phe) is an amino acid with a nonpolar aromatic side chain. Phe has one of the highest hydrophobicity scales, which drives it into nonpolar environments, e.g. the hydrocarbon core of phospholipid membranes [1-6]. On one hand, this offers a great therapeutic potential. The incorporation of Phe residues has indeed demonstrated to drastically increase the potency of antimicrobial peptides plausibly by enabling stronger interaction with the bacterial cell envelope [7, 8]. On the other hand, the propensity of Phe for the membrane hydrocarbon core together with its tendency to cluster has been implicated in metabolic and neurological disorders [6, 9-14]. Phe residues have been

\footnotetext{
* Correspondence: valeriat@yorku.ca

Department of Chemistry, York University, Toronto, ON M3J 1P3, Canada
}

identified as major amyloidogenic sites in proteins and peptides involved with proteopathies, e.g. Alzheimer's and prion diseases, type II diabetes, etc. [14]. Phe is also the only amino acid capable of self-assembling into supramolecular structures with amyloid morphology [6, 9-13]. Interactions of these structures with membrane phospholipids have been suggested to cause cell toxicity, in particular in phenylketonuria, by perturbing the phospholipid packing and compromising the membrane structural integrity $[9,10]$. This has thus put Phe/membrane interactions into the focus of renewed research interest over the recent years $[5,6,12,13,15,16]$.

A number of studies have examined Phe self-assembly and interactions with model phospholipid membranes $[1,5,6,9-13,15-17]$. It has been reported that Phe is capable of intercalating into the membrane through small-scale packing defects $[1,5,15]$, forming Phe/

(c) The Author(s). 2018 Open Access This article is distributed under the terms of the Creative Commons Attribution 4.0 International License (http://creativecommons.org/licenses/by/4.0/), which permits unrestricted use, distribution, and 
phospholipid complexes [15-17], self-assembling into tubular pore- and needle-like structures piercing through the membrane $[6,11,13]$, and depositing fibrils onto the membrane surface $[9,12]$. The depth of Phe localization in the membrane has been found to vary significantly, from lying nearly flat at the membrane surface to being embedded into the hydrocarbon core $[1,5,12,16]$. These observations point towards a multitude of modes Phe can use when interacting with membranes. An account of possible Phe interaction modes is currently available in the literature $[1,5,6,9-13,15-17]$. However, what remains to be further investigated is precise molecular level details of the initial Phe/membrane interaction, e.g. Phe intercalation parameters, the effect of hydration, counterions, etc., and how they may be predetermining the cascade of events to follow. Such knowledge is crucial for the advancement of our understanding of disease mechanisms and for the development of new approaches to the design of therapeutic agents.

In this study, we used Langmuir monolayers made up of a single phospholipid as the simplest membrane models. The initial interaction of membranes with Phe was modeled by injecting Phe into the aqueous phase underneath the monolayer. The advantage of monolayers as membrane models is that they offer precise control over the lateral pressure (surface pressure, in monolayer terms) and area per phospholipid molecule. This provides a unique opportunity to measure the expansion of membrane area induced by a biomolecule dissolved in the aqueous phase, e.g. Phe, in the constant pressure insertion assay [18-20]. Area expansion data can then be used to estimate biomolecule intercalation parameters $[18,19]$. In particular, in our study, area expansion data afforded determining the Phe intercalation area, $A_{\text {Phe }}$. Augmented with epifluorescence microscopy, the assay also allowed to identify the membrane environment involved with Phe intercalation. Based on $A_{\text {Phe }}$ values, Phe intercalation modes were assessed. Two modes have been found to operate in model membranes, one of them plausibly involving a counterion.

\section{Methods}

\section{Materials}

Both phospholipids, 1,2-dipalmitoyl-sn-glycero-3-phosphoethanolamine (DPPE) and 1,2-dipalmitoyl-sn-glycero-3-phosphocholine (DPPC), and the fluorescent probe, 1,2-dioleoyl-sn-glycero-3-phosphoethanolamine-N-(Lissamine Rhodamine B Sulfonyl) labeled at the headgroup (DOPE-Rh), were purchased from Avanti Polar Lipids, Inc. The phospholipids were $>99 \%$ pure and used without further purification. Phenylalanine (99\%) and phosphate buffered saline (PBS) were purchased from Sigma. Solvents used were of HPLC grade from Fisher Scientific. Deionized water with $18.2 \mathrm{M} \Omega \bullet \mathrm{cm}$ resistivity was produced by a Milli-Q Synthesis water purification system (EMD Millipore, MA).

\section{Model membranes}

Model membranes were prepared by spreading phospholipids, DPPE or DPPC, from chloroform solutions at the air/water interface in a KSV2000SP Langmuir trough (KSV Instruments Ltd., Finland). For spreading solutions, a phospholipid concentration of $\sim 1 \mathrm{mg} / \mathrm{mL}$ was used. Spreading volume was adjusted such that model membranes were formed over the most of the trough area to make available as much of membrane surface as possible for interactions with Phe. To form model membranes, phospholipids were compressed to a packing density similar to that in a biological membrane. While the mean molecular area, $A$, reduced upon compression, the surface pressure, $\pi$, was recorded as $\pi-A$ isotherms. A filter paper Wilhelmy plate was used to measure $\pi$ to an accuracy of $0.1 \mathrm{mN} / \mathrm{m}$. The trough was thermostated to maintain a physiologically relevant temperature of $37 \pm 1{ }^{\circ} \mathrm{C}$ [21].

\section{Area expansion measurements}

To study Phe-induced expansion of model membranes, the constant pressure insertion assay was used [18-20]. In this assay, monolayers were compressed to an area of $\sim 52$ and $\sim 65 \AA^{2} /$ molecule for DPPE and DPPC, respectively, which corresponds to their typical packing densities in a biological membrane [22]. The monolayer pressure, $\pi$, was then set to maintain constant by the trough electronic feedback device controlling the movement of two barriers whereas the area per phospholipid molecule, $A$, was allowed to change. After $20 \mathrm{~min}$ to allow for a monolayer to equilibrate, Phe was injected underneath the monolayer and let to interact for $2 \mathrm{~h}$. The Phe-induced area expansion was recorded over time, $t$, as $A-t$ isotherms.

\section{Epifluorescence microscopy}

For the EFM imaging of model membranes, the Langmuir trough was interfaced with an upright Nikon Eclipse FN1 epifluorescence microscope. To enable the imaging, phospholipid spreading solutions were labeled with $\sim 0.5 \mathrm{~mol} \%$ of fluorescent probe, DOPE-Rh. Imaging was performed with the Nikon TRITC HYQ filter combination $545 \mathrm{CWL}$ excitation filter, 570LP dichroic mirror and $620 \mathrm{CWL}$ barrier filter) through a $10 \mathrm{x}$ objective. The images were captured by a CCD camera directly onto a computer screen. Image processing and analysis was performed with the Simple PCI 6 software (Compix Inc., PA). 


\section{Results}

Phe is known to have a propensity for partitioning in the hydrocarbon core of phospholipid membranes [1, 2]. When injected underneath model membranes, Phe caused an expansion of monolayer area in the constant pressure insertion assay. However, the Phe-induced area expansion strongly depended on Phe concentration. At Phe concentrations below $\sim 0.25 \mathrm{mM}$, no expansion of monolayer area was detected. Phe-induced area expansion became noticeable above $\sim 1.25 \mathrm{mM}$ of Phe (see Additional file 1: Figure 1S). This concentration-dependent expansion of monolayer area indicates that, beyond a certain concentration threshold, Phe molecules collectively intercalate into the model membrane and may perturb phospholipid packing. To examine the effect of Phe intercalation on model membranes, the data presented below were obtained at $\sim 2.5 \mathrm{mM}$ of Phe. It is worth noting that this concentration is well within pathologic Phe levels $[5,9,10,23]$.

\section{In-situ imaging of Phe intercalation}

The effect of Phe intercalation on model membranes was first visualized with EFM. According to a recent study [5], Phe is likely to intercalate into the membrane through small-scale packing defects in the liquid-disordered $\left(\mathrm{L}_{\mathrm{d}}\right)$ phase. Hence, in this study, we used DOPE-Rh as the fluorescent probe to image the $\mathrm{L}_{\mathrm{d}}$ phase. The probe is known to preferentially partition in this phase [24]. The $L_{d}$ phase thus appears stained red in the images (in Figs. 1 and 2 discussed below).

Figure 1 shows images of a DPPE monolayer before and after Phe injection. The monolayer was compressed to an area of $\sim 52 \AA^{2} /$ molecule and kept at a constant pressure. Dark field in image A captured before Phe injection indicates that, at $52 \AA^{2} /$ molecule, DPPE is predominantly in the gel state. The gel phase appears dark in EFM images because the fluorescent probe, DOPE- $\mathrm{Rh}$, is excluded from it [24]. DPPE typically forms the $L_{\beta}$ gel [25]. In the $L_{\beta}$ gel, the hydrocarbon chains of DPPE are untilted as depicted in the schematic in Fig. 1c. Red fluorescent domains in the image suggest that the fluid $L_{d}$ phase is also present (see the same image with a $1.5 \mathrm{x}$ magnification in Additional file 1: Figure $2 \mathrm{~S}$ (image A) ). In the $L_{d}$ phase, the hydrocarbon chains are disordered. The $\mathrm{L}_{\mathrm{d}}$ phase is depicted stained red in the schematic in Fig. 1c.

The disorder of $\mathrm{L}_{\mathrm{d}}$ phase allows dissolved biomolecules to interact with membranes, which makes this phase the most biologically relevant. Indeed, the comparison of images in Fig. 1 shows a noticeable expansion of red $L_{d}$ phase domains $2 \mathrm{~h}$ after Phe injection, plausibly due to Phe intercalation. This is depicted in the schematic in Fig. 1c. Although the monolayer morphology remains essentially the same, the area fraction of fluorescent $L_{d}$ phase domains in image $B$ has increased by $\sim 10 \%$ as compared to image A. Moreover, when
A

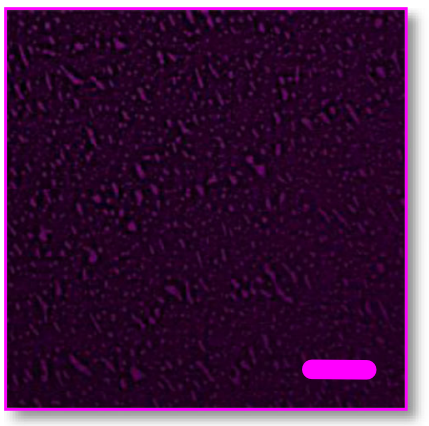

C

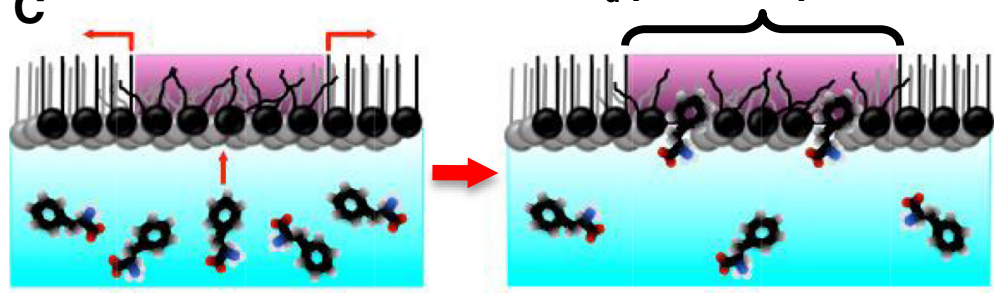

B

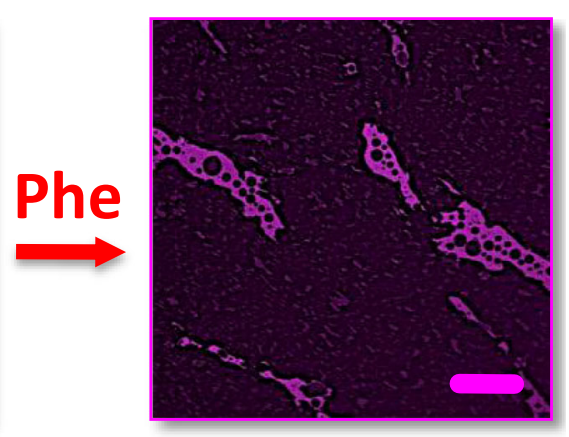

$L_{\mathrm{d}}$ phase expansion
Fig. 1 Phe intercalation into the DPPE model membrane. EFM images show the membrane morphology before (a) and 2 h after (b) Phe injection. Red staining indentifies the membrane $L_{d}$ phase. Phe injection causes the area of $L_{d}$ phase to expand plausibly due to the intercalation of Phe, which is depicted in the schematic (c). Images are for a DPPE monolayer initially at an $A$ of $\sim 52 \AA^{2} / \mathrm{molecule}$ on PBS at $37{ }^{\circ} \mathrm{C}$. Scale bar is $50 \mu \mathrm{m}$ 
A

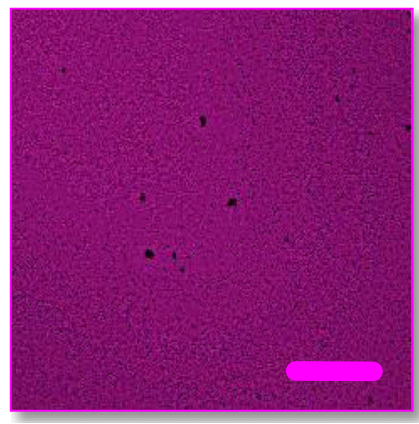

B

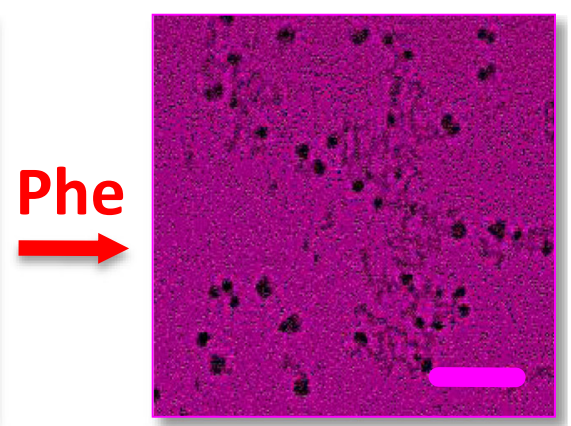

Fig. 2 Phe intercalation into the DPPC model membrane. EFM images show the membrane morphology before (a) and $2 \mathrm{~h}$ after (b) Phe injection. Red staining indentifies the membrane $L_{d}$ phase. Images are for a DPPC monolayer initially at an $A$ of $\sim 65 \AA^{2} / \mathrm{molecule}$ on PBS at $37^{\circ} \mathrm{C}$. Scale bar is $50 \mu \mathrm{m}$

expanding, some of the $\mathrm{L}_{\mathrm{d}}$ domains appear to merge in trails propagating through the gel phase (image B in Fig. 1 ). Such $L_{d}$ phase trails started to appear in DPPE monolayers $\sim 1 \mathrm{~h}$ after Phe injection and were highly reproducible. These observations thus confirm that Phe intercalates into the model DPPE membrane primarily through the $\mathrm{L}_{\mathrm{d}}$ phase. (To rule out any artifacts, control measurements were performed with DPPE alone. In these experiments, a DPPE monolayer was compressed to the same area of $52 \AA^{2} /$ molecule and kept for $2 \mathrm{~h}$ at a constant pressure without Phe injection. EFM imaging showed a contraction of red $\mathrm{L}_{\mathrm{d}}$ phase domains over time (see Additional file 1: Figure 2S), which is completely opposite to the trend seen in Fig. 1b.)

As compared to DPPE, the effect of Phe injection on model DPPC membranes is more intricate. Figure 2 shows images of a DPPC monolayer before and after Phe injection. The monolayer was compressed to an area of $\sim 65 \AA^{2} /$ molecule. The latter value, although larger than that for DPPE, corresponds to the typical DPPC packing density in a biological membrane [22]. As seen in EFM images in Fig. 2, Phe induces striking changes in the DPPC monolayer morphology. Tiny dark gel domains in a sea of red fluorescent fluid phase in image A indicate a two-phase coexistence in the DPPC monolayer before Phe injection. Starkly different from the signature kidney-shaped domains usually observed in DPPC monolayers at $20{ }^{\circ} \mathrm{C}$, these tiny gel phase domains appear to predominate in the morphology of DPPC monolayers at elevated temperatures as has been visualized by other imaging techniques [26, 27]. Image B captured $2 \mathrm{~h}$ after Phe injection, reveals that both the number and size of the dark gel domains significantly increases. This points towards a massive nucleation and growth of the DPPC gel phase, which is likely triggered by Phe intercalation. A similar trend was reported for $\mathrm{DPPC} /$ Phe interactions at $20{ }^{\circ} \mathrm{C}$ [5]. Interestingly, the effect of Phe on DPPC monolayers appears to contrast with that observed for DPPE, which might imply a difference in Phe intercalation parameters. A series of measurement was thus performed to determine the intercalation area of Phe for both DPPC and DPPE membranes as discussed below.

\section{Phenylalanine-induced expansion of model membranes}

The Phe-induced expansion of membrane area was also measured with the Langmuir balance. The measurements were performed simultaneously with EFM imaging by using the constant pressure insertion assay protocol [18-20]. A second set of area expansion measurements without EFM was performed as well to avoid labeling the model membranes with DOPE-Rh. These measurements were designed as a control series to assess the effect of fluorescent probe, if any, on Phe/membrane interactions. Both series of measurements produced identical results thus indicating that the use of DOPE-Rh did not give rise to any artifacts. Figure 3 shows typical $A-t$ isotherms from the insertion assays. As seen in the figure, Phe injection caused the area per phospholipid molecule, $A$, to increase over time. For both monolayers, $A-t$ isotherms leveled off to a steady-state value of $A \sim 1.5 \mathrm{~h}$ after Phe injection as shown in Fig. 3.

The DPPE monolayer, initially at $52 \AA^{2} /$ molecule, exhibits a $\sim 7 \AA^{2}$ increase in $A$ when its interactions with Phe reach the steady state. This Phe-induced area expansion is denoted as $\Delta A$ in Fig. 3. By relating $\Delta A$ to the initial area, $A$ (before Phe injection), the relative area expansion, $\triangle A / A$, can be estimated. For DPPE, $\triangle A / A$ expressed in $\%$ amounts to $\sim 12 \%$. The latter value correlates well with the $\sim 10 \%$ increase of the area fraction of fluorescent $\mathrm{L}_{\mathrm{d}}$ phase observed in the EFM images in Fig. 1. For the DPPC monolayer, somewhat larger $\triangle A$ values were recorded. When related to the initial DPPC 


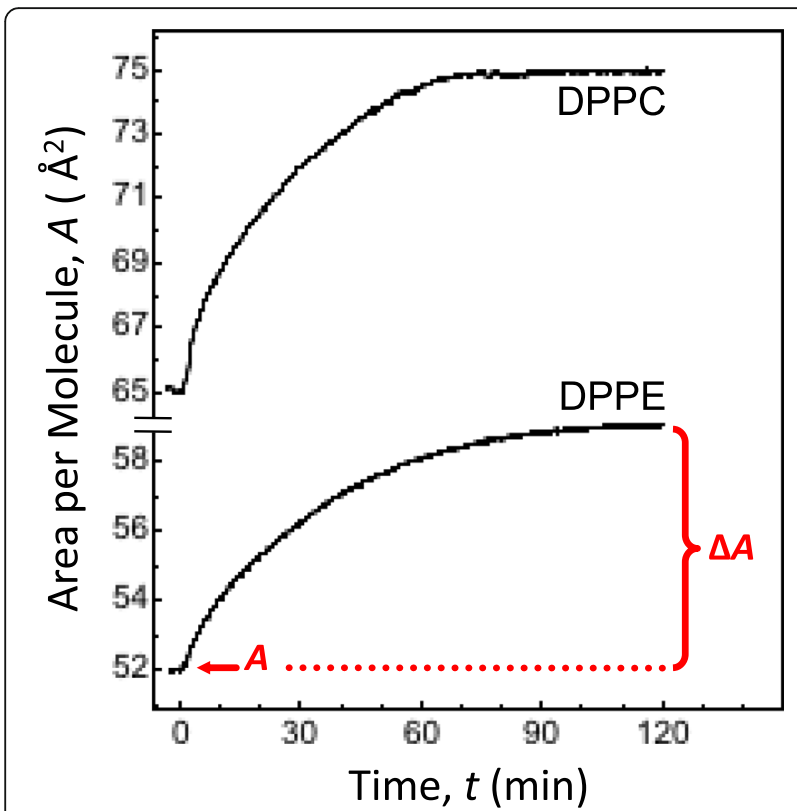

Fig. 3 Typical area expansion $A-t$ isotherms from insertion assays. The isotherms are for a DPPE and DPPC monolayer initially at an $A$ of $\sim 52$ and $\sim 65 \AA^{2} /$ molecule, respectively. Time $t=0$ in the isotherms corresponds to Phe injection point. $\Delta A$ indicates the Pheinduced area expansion in the steady state. By relating $\Delta A$ to $A$, the relative area expansion, $\triangle A / A$, is obtained. The data are for monolayers on PBS at $37^{\circ} \mathrm{C}$

area of $65 \AA^{2} /$ molecule, they yielded a value of $\sim 15 \%$ for $\triangle A / A$. The $A-t$ data thus confirm that both model membranes undergo a noticeable area expansion upon Phe injection. This suggests that Phe is capable of intercalating both DPPE and DPPC packing.

\section{Phenylalanine intercalation area}

A number of studies have demonstrated that the relative area increase, $\triangle A / A$, can be used to calculate the area a biomolecule takes when inserting in a model membrane $[18,19]$. Based on the simple Boltzmann factor [18], the relationship between $\triangle A / A$ and Phe intercalation area, $A_{\text {Phe }}$, can be written as.

$\ln (\triangle A / A) \approx-\pi A_{\text {Phe }} / k T(1)$.

Here, $\pi$ is the surface pressure, $k$ is the Boltzmann constant and $T$ is the temperature [18]. As follows from eq. 1 , a semilogarithmic plot of $\triangle A / A$ as a function of $\pi$ should yield a regression line with a negative slope $-A_{\text {Phe }} / k T$. From the slope of the line, a value for $A_{\text {Phe }}$ can then be determined.

To enable the use of eq. 1 , a set of $\triangle A / A$ values was obtained over a wider range of $A$ centered at 52 and $65 \AA^{2}$ / molecule for DPPE and DPPC monolayer, respectively. For DPPE, $\triangle A / A$ values were measured for monolayers with the initial area in a range of $48-56 \AA^{2} /$ molecule. For DPPC, the measurements were performed over a range of 60-70 $\AA^{2} /$ molecule. For the measurements, the constant pressure insertion assay protocol was used. Values of $\pi$ corresponding to the selected $A$ ranges span from $\sim 13$ to $25 \mathrm{mN} / \mathrm{m}$ (For correlation between $\pi$ and $A$ values, see Additional file 1: Figure $3 \mathrm{~S}$ ). Figure 4 shows the $\triangle A / A$ data for both monolayers as a function of $\pi$. As seen in Fig. 4a, Phe caused noticeable expansion in both monolayers over the entire range of selected $A$ and $\pi$ values. Somewhat larger relative area expansion was observed for DPPC monolayers on PBS. For comparison, Fig. 4 also displays the $\triangle A / A$ data for both monolayers on water, which is discussed in more detail in the section 3.4 below. All monolayers showed a decrease in the Phe-induced $\triangle A / A$ with increasing $\pi$ (Fig. 4a). Phe ceased to cause the expansion of monolayers area at a $\pi$ of $\sim 32 \mathrm{mN} / \mathrm{m}$ (see Additional file 1: Figure 4S).

To determine the Phe intercalation area, $A_{\text {Phe, the }} \Delta A$ / $A$ data are plotted on a semilogarithmic scale as a function of $\pi$ in Fig. 4b. As seen in the figure, all datasets produce linear regressions with a negative slope as predicted by eq. 1 . This indicates that Phe intercalates in the model membranes with a constant $A_{\text {Phe }}[18,19]$. However, there is a marked difference in the regression slope between the dataset for DPPC on PBS and other monolayers. Based on eq. 1, this suggests two different $A_{\text {Phe }}$ values. Indeed, from the slopes of linear fits in Fig. $4 \mathrm{~b}$, the intercalation areas of $33 \pm 2$ and $48 \pm 3 \AA^{2}$ were obtained for Phe. The former value correlates with the area of $\sim 28 \AA^{2}$ estimated for a molecule of aromatic amines [17], whereas the latter value points towards a larger Phe species (e.g. a sodiated Phe complex [28, 29]) intercalating in the DPPC monolayer from PBS as discussed below.

\section{Discussion}

\section{Counterion in Phe intercalation}

Among the aromatic amino acids, Phe exhibits the largest conformational variety [30-32]. Phe conformers mainly differ in the degree of alanyl chain folding with respect to the Phe ring [30]. This conformational variety might allow Phe to use different modes when binding to surfaces and interfaces. In particular, Phe is often viewed as stretched along the surface normal with the Phe ring embedded in the nonpolar phase and its charged groups facing the polar phase $[4,5,15-17,33]$. Based on the data available in the literature, the area per Phe molecule in such a conformation ranges from 24 to $33 \AA^{2}[4,17$, 33]. The latter correlates well with the $A_{\text {Phe }}$ value of $33 \pm 2 \AA^{2}$ obtained in our study. This value is thus consistent with a Phe monomer intercalating into the model membranes by inserting the phenyl ring into the membrane hydrocarbon core nearly normal to the membrane surface with its headgroup positioned in the aqueous region of the interface as predicted by earlier studies 

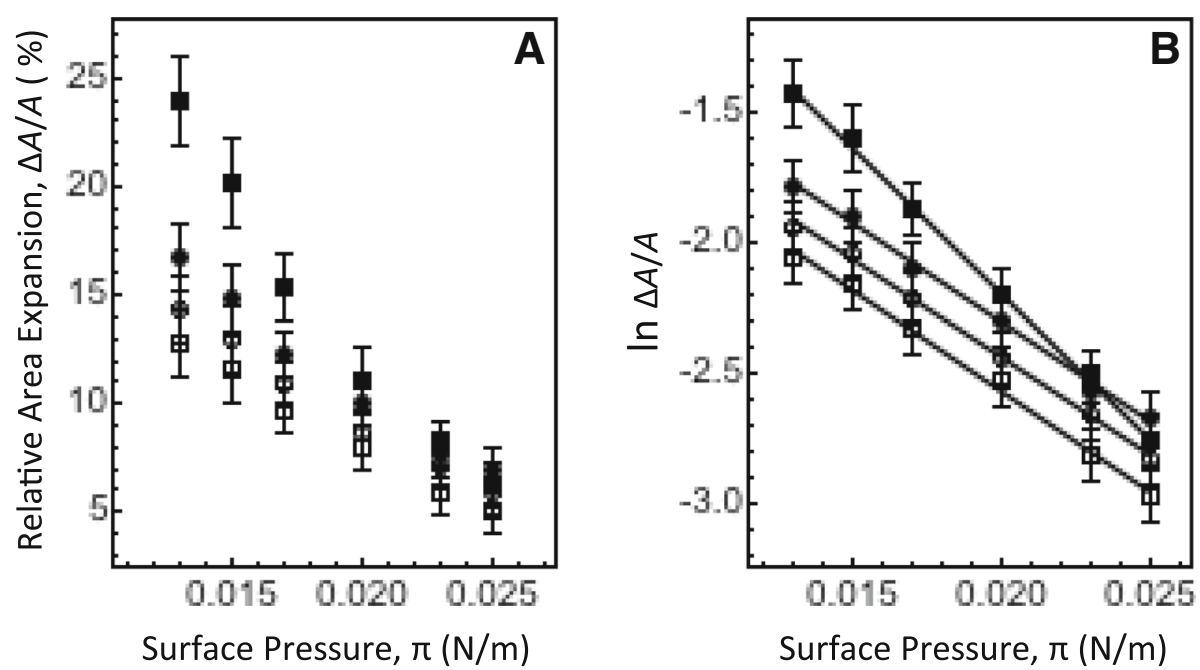

Fig. 4 Phe insertion assay data analysis. a Steady-state $\triangle A / A$ as a function of $\pi$ for DPPE and DPPC monolayers on water (open circles and squares, respectively) and PBS (filled circles and squares, respectively). b Steady-state $\Delta A / A$ as a function of $\pi$ on a semilogarithmic scale. Solid lines are linear fit to the data. From the slope of linear fits, two different values for $A_{\text {phe }}$ were obtained. For the DPPC monolayer on PBS (filled

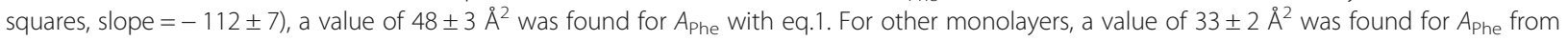
the virtually identical slopes of $-77 \pm 2,-76 \pm 3$ and $-78 \pm 2$. The data are for DPPE and DPPC monolayers initially at $52 \pm 4$ and $65 \pm 5 \AA^{2} /$ molecule, respectively

$[5,15]$. Figure 5a schematically depicts this mode of Phe intercalation. Found at the higher range of reported Phe areas, the value of $33 \pm 2 \AA^{2}$ may also suggest that the headgroup of Phe remains at least partially hydrated. In fact, hydration of Phe with two water molecules, ammonium- and carboxyl-bound, has been shown to present a stabilizing structural element in the amino acid molecule [34]. The two water dipoles line up favorably with the charged groups and help maintaining the relative positions of the phenyl ring and alanyl chain in the molecule [34]. However, this mode appears to be valid primarily for Phe intercalation into model membranes formed on water, that is when no counterions are present. The difference in the $A_{\text {Phe }}$ values obtained for monolayers on PBS suggests that the addition of saline may significantly affect the mode of Phe intercalation, in particular for DPPC.

The rather large value of $A_{\text {Phe }}=48 \pm 3 \AA^{2}$ obtained for the DPPC monolayer on PBS points towards the intercalation of Phe either in a different conformation or as a larger species. In aqueous solutions, aromatic $\pi$ - rings have been shown to successfully compete with water in binding to alkali metal cations and stably retain them in a $\pi$ - cation complex structure [28, 29]. In particular, $\mathrm{Na}^{+}$cation (present in PBS at a concentration of $130 \mathrm{mM}$ ) has been suggested to simultaneously interact
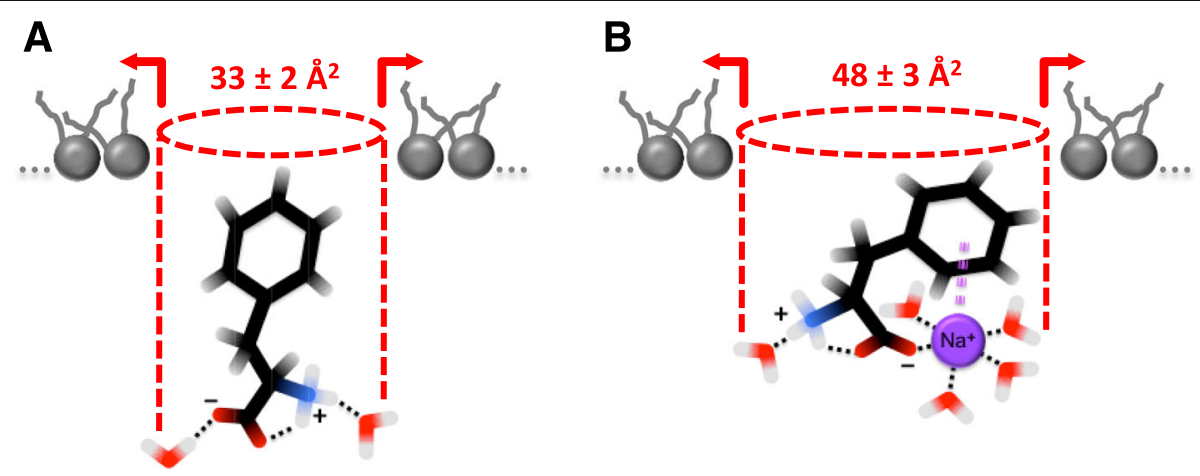

Fig. 5 Two modes of Phe intercalation into model membranes. a The intercalation mode involving a Phe monomer. In this mode, the phenyl ring inserts between phospholipid molecules in the $L_{d}$ phase nearly normal to the membrane surface, which is consistent with the $A_{\text {phe }}$ value of $33 \pm 2 \AA^{2}$. Phe may retain its hydration water molecules as shown with dotted traces. $\mathbf{b}$ The intercalation mode involving a $\pi-$ cation complex. In the complex, a cation, e.g. $\mathrm{Na}^{+}$, links the Phe ring and carboxylate group through its first coordination shell [25], which is shown with dotted traces. This is likely to confine the Phe ring in a titlted conformation to the membrane headgroup region, thereby taking up a larger area of $48 \pm 3 \AA^{2}$ as indicated with dashed lines 
with the $\pi$-electron cloud and carboxylate group of zwitterionic Phe $[28,29]$. In this $\mathrm{Na}^{+}(\mathrm{Phe})$ complex structure, $\mathrm{Na}^{+}$cation sheds two water molecules of its first coordination shell to link the phenyl ring and carboxylate group via a bidentate geometry [29]. This is drawn schematically in Fig. 5b. Such a complexation with $\mathrm{Na}^{+}$ is likely to anchor the Phe ring to the aqueous phase and encourage the folding of alanyl chain. Instead of stretching into the hydrocarbon region, the sodiated Phe ring may thus adopt a tilted conformation in the headgroup region, thereby taking up a larger area when inserting in the model membrane. In fact, a study by Yang et al. [33] suggests that mere tilting of Phe ring with respect to the surface normal causes the area occupied by the amino acid molecule to increase. Moreover, $\mathrm{Na}^{+}$will also add to the area of sodiated Phe complex. A value of $\sim 2.3 \AA$ has been reported for the radius of hydrated $\mathrm{Na}^{+}$cation [35], which corresponds to an area of $\sim 16.6 \AA^{2}$. The latter appears to correlate with the $15 \AA^{2}$ increase in $A_{\text {Phe }}$ (from $33 \pm 2 \AA^{2}$ on water to $48 \pm 3 \AA^{2}$ on PBS) observed for DPPC in our study, which is consistent with Phe dragging along $\mathrm{Na}^{+}$and intercalating into the membrane as a $\mathrm{Na}^{+}$(Phe) complex.

\section{Biological implications of Phe intercalation}

The difference between the $A_{\text {Phe }}$ values obtained for DPPE and DPPC model membranes in our study suggests that Phe is indeed capable of using at least two distinct modes of intercalation. This may have implications for a number of processes triggered by elevated extracellular Phe levels. In particular, the intercalation of Phe involving a counterion, e.g. $\mathrm{Na}^{+}$depicted in Fig. 5b, may have a role in the accumulation of amyloid-like Phe deposits at the membrane surface under pathological conditions. Of the most abundant membrane phospholipids, DPPC is located primarily in the outer leaflet of the membrane exposed to the extracellular fluid [36]. Phe intercalation, which is feasible at elevated Phe levels [5, 9-12], is thus likely to use the mode involving a counterion as depicted in Fig. 5b for the DPPC model membrane. Elevation in extracellular Phe can also induce the self-assembly of Phe into amyloid fibrils $[9,10]$. This self-assembly is believed to occur through the parallel $\pi$-stacking of Phe rings $[9,11,13]$. Although counterions have been shown to drive the Phe stacking by stabilizing the self-assembled amyloid structures [13], the exact mechanism of interactions involved with the deposition of Phe fibrils onto the membrane surface remains largely unclear. The findings of this study enable us to hypothesize that the intercalation of Phe in a titled configuration exposing the sodiated Phe ring may in fact provide nucleation and/or docking sites for the self-assembly and anchoring of Phe amyloid fibrils at the membrane surface.

\section{Conclusions}

Phe is capable of intercalating through the $L_{\mathrm{d}}$ phase into both DPPE and DPPC model membranes. Two modes of intercalation are likely to be involved. The modes differ by the $A_{\text {Phe }}$ area taken by each Phe molecule upon intercalation. The area of $A_{\text {Phe }} 33 \pm 2 \AA^{2}$ is consistent with a Phe monomer intercalating into membrane by inserting the phenyl ring nearly normal to the membrane surface. This mode operates in DPPE membranes in both absence and presence of counterions. For DPPC membranes however, counterions appear to alter the mode of Phe intercalation. The value of $A_{\mathrm{Phe}}=48 \pm 3 \AA^{2}$ suggests that, from saline, Phe tends to intercalate into DPPC membranes as a larger species, e.g. a $\mathrm{Na}^{+}$(Phe) complex. Complexation with $\mathrm{Na}^{+}$is likely to anchor Phe ring to the aqueous phase, which may provide nucleation sites for Phe self-assembly. These findings can be instrumental in understanding various membrane processes triggered by interactions with Phe, in particular the early events leading to the deposition of Phe amyloid fibrils onto the membrane surface.

\section{Additional file}

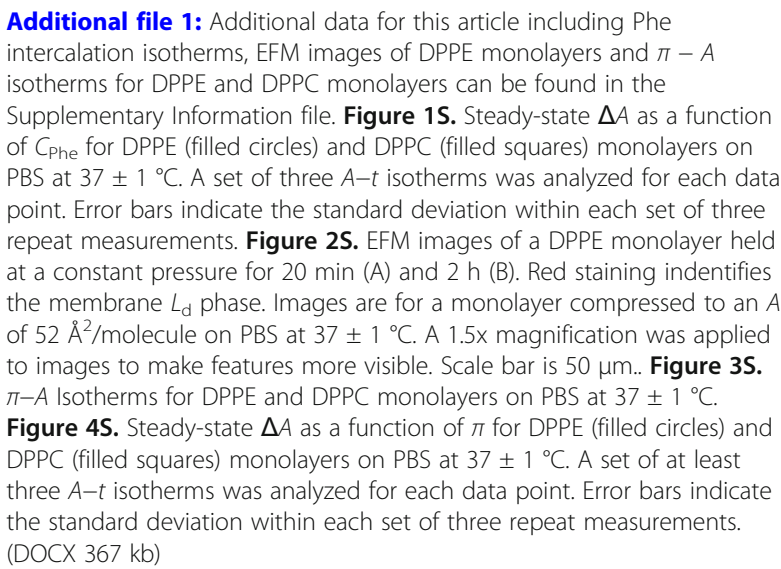

\section{Abbreviations}

DOPE-Rh: 1,2-dioleoyl-sn-glycero-3-phosphoethanolamine-N-(Lissamine Rhodamine B Sulfonyl); DPPC: 1,2-dipalmitoyl-sn-glycero-3-phosphocholine; DPPE: 1,2-dipalmitoyl-sn-glycero-3-phosphoethanolamine;

EFM: epifluorescence microscopy; PBS: phosphate buffered saline; Phe: Phenylalanine

\section{Acknowledgements}

Not applicable.

\section{Funding}

The authors thank the Natural Sciences and Engineering Research Council of Canada and Canada Foundation for Innovation for the financial support to this study. The support was used to purchase instruments and materials for the study. The funding body played no role in the design of the study, collection, analysis and interpretation of the data, and in writing the manuscript. 


\section{Availability of data and materials}

All data generated or analyzed during this study are included in this published article and in its Supplementary Information file.

\section{Authors' contributions}

PA carried out the experiments and collected the data. VT designed the study, built the experimental set-up, analyzed the data and drafted the manuscript. Both authors approved the final version of the manuscript.

\section{Ethics approval and consent to participate}

Not applicable.

\section{Consent for publication}

Not applicable.

\section{Competing interests}

The authors declare that they have no competing interests.

\section{Publisher's Note}

Springer Nature remains neutral with regard to jurisdictional claims in published maps and institutional affiliations.

Received: 26 April 2018 Accepted: 31 October 2018 Published online: 15 November 2018

\section{References}

1. MacCallum JL, Bennett WFD, Tieleman DP. Distribution of amino acids in a lipid bilayer from computer simulations. Biophys J. 2008;94:3393.

2. Morita M, Katta AVSKM, Ahmad S, Mori T, Sugita Y, Mizuguchi K. Lipid recognition propensities of amino acids in membrane proteins from atomic resolution data. BMC Biophys. 2011:4:21.

3. Griffith EC, Vaida V. Ionization state of L-phenylalanine at the air-water interface. J Am Chem Soc. 2012:135:710.

4. Onorato RM, Yoon AP, Lin JT, Somorjai GA. Adsorption of amino acids and dipeptides to the hydrophobic polystyrene interface studied by SFG and QCM: the special case of phenylalanine. J Phys Chem C. 2012;116:9947.

5. Griffith EC, Perkins RJ, Telesford DM, Adams EM, Cwiklik L, Allen HC, Roeselová M, Vaida V. Interaction of L-phenylalanine with a phospholipid monolayer at the water-air interface. J Phys Chem B. 2015:1 19:9038.

6. Perkins R, Vaida V. Phenylalanine increases membrane permeability. J Am Chem Soc. 2017;139:14388

7. Konai MM, Ghosh C, Yarlagadda V, Samaddar S, Haldar J. Membrane active phenylalanine conjugated lipophilic norspermidine derivatives with selective antibacterial activity. J Med Chem. 2014:57:9409.

8. Shahmiri M, Cornell B, Mechler A. Phenylalanine residues act as membrane anchors in the antimicrobial action of Aurein 1.2. Biointerphases. 2017:12: $05 \mathrm{G} 605$.

9. Adler-Abramovich L, Vaks L, Carny O, Trudler D, Magno A, Caflisch A, Frenkel D, Gazit E. Phenylalanine assembly into toxic fibrils suggests amyloid etiology in phenylketonuria. Nat Chem Biol. 2012;8:701.

10. Singh V, Rai RK, Arora A, Sinha N, Thakur AK. Therapeutic implications of Lphenylalanine aggregation mechanism and its modulation by Dphenylalanine in phenylketonuria. Sci Rep. 2014:4:1.

11. German HM, Uyaver S, Hansmann UHE. Self-assembly of phenylalaninebased molecules. J Phys Chem B. 2015:119:1609.

12. Sankaranarayanan K. Fibrils of phenylalanine adsorbed to Langmuir-Blodgett films: role of lipids. Soft Materials. 2015:13:219.

13. Do TD, Kincannon WM, Bowers MT. Phenylalanine oligomers and fibrils: the mechanism of assembly and the importance of tetramers and counterions. J Am Chem Soc. 2015;137:10080

14. Chiti F, Dobson CM. Protein misfolding, amyloid formation, and human disease: a summary of progress over the last decade. Annu Rev Biochem. 2017;86:27.

15. Rosa AS, Cutro AC, Frías MA, Disalvo EA. Interaction of phenylalanine with DPPC model membranes: more than a hydrophobic interaction. J Phys Chem B. 2015:119:15844

16. Cutró AC, Hollmann A, Cejas J, Maturana P, Disalvo EA, Frías MA. Phenylalanine interaction with lipid monolayers at different $\mathrm{pHs}$. Colloids Surf B. 2015;135:504
17. Petelska AD, Naumowicz M, Figaszewski ZA. The equilibrium of phosphatidylcholine-amino acid system in monolayer at the air/wate interface. Cell Biochem Biophys. 2011;60:155.

18. Boguslavsky V, Rebecchi M, Morris AJ, Jhon DY, Rhee SG, Mc Laughlin S. Effect of monolayer surface pressure on the activities of phosphoinositidespecific phospholipase C- $\beta 1,-\gamma 1$, and - -1 . Biochemistry. 1994;33:3032.

19. Hanakam F, Gerisch G, Lotz S, Alt T, Seelig A. Binding of hisactophilin I and II to lipid membranes is controlled by a pH-dependent myristoyl-histidine switch. Biochemistry. 1996:35:11036.

20. Ishitsuka Y, Pham DS, Waring AJ, Lehrer RI, Lee KYC. Insertion selectivity of antimicrobial peptide protegrin-1 into lipid monolayers: effect of head group electrostatics and tail group packing. Biochim Biophys Acta. 2006;1758:1450.

21. Peetla C, Labhasetwar V. Biophysical characterization of nanoparticleendothelial model cell membrane interactions. Mol Pharm. 2008:5:418.

22. Leekumjorn S, Sum AK. Molecular simulation study of structural and dynamic properties of mixed DPPC/DPPE bilayers. Biophys J. 2006;90:3951.

23. Hanley WB. Adult phenylketonuria. Am J Med. 2004;117:590.

24. Klymchenko AS, Kreder R. Fluorescent probes for lipid rafts: from model membranes to living cells. Chem Biol. 2014;16:97.

25. McIntosh TJ. Differences in hydrocarbon chain tilt between hydrated phosphatidylethanolamine and phosphatidylcholine bilayers. Biophys J. 1980;29:237

26. Toimil P, Prieto G, Miñones J Jr, Sarmiento F. A comparative study of FDPPC/DPPC mixed monolayers. Influence of subphase temperature on $\mathrm{F}$ DPPC and DPPC monolayers. Phys Chem Chem Phys. 2010;12:13323.

27. Zuo YY, Chen R, Wang X, Yang J, Policova Z, Neumann AW. Phase transitions in dipalmitoylphosphatidylcholine monolayers. Langmuir. 2016;32:8501.

28. Siu FM, Ma NL, Tsang CW. Cation- $\pi$ interactions in sodiated phenylalanine complexes: is phenylalanine in the charge-solvated or zwitterionic form? J Am Chem Soc. 2001;123:3397.

29. Costanzo F, Della Valle RG, Barone V. MD simulation of the $\mathrm{Na}^{+}$-phenylalanine complex in water: competition between cation- $\pi$ interaction and aqueous solvation. J Phys Chem B. 2005:109:23016.

30. Lee Y, Jung J, Kim B, Butz P, Snoek LC, Kroemer RT, Simons JP. Alanyl side chain folding in phenylalanine: conformational assignments through ultraviolet rotational band contour analysis. J Phys Chem A. 2004;108:69.

31. Kaczor A, Reva ID, Proniewicz LM, Fausto R. Importance of entropy in the conformational equilibrium of phenylalanine: a matrix-isolation infrared spectroscopy and density functional theory study. J Phys Chem A. 2006;110:2360.

32. Shimozono Y, Yamada K, Ishiuchi SI, Tsukiyama K, Fujii M. Revised conformational assignments and conformational evolution of tyrosine by laser desorption supersonic jet laser spectroscopy. Phys Chem Chem Phys. 2013;15:5163.

33. Yang S, Lim H, Park EH, Kim Y, Min YH, Lee HS, Kim S, Lee H. Comparison and contrast analysis of adsorption geometries of phenylalanine versus tyrosine on Ge(100): effect of nucleophilic group on the surface. J Phys Chem C. 2012;116:25840

34. Gao B, Wyttenbach T, Bowers MT. Hydration of protonated aromatic amino acids: phenylalanine, tryptophan, and tyrosine. J Am Chem Soc. 2009;131:4695.

35. Yang KL, Yiacoumi S, Tsouris C. Monte Carlo simulations of electrical double-layer formation in nanopores. J Chem Phys. 2002;117:8499.

36. Ikeda M, Kihara A, Igarashi Y. Lipid asymmetry of the eukaryotic plasma membrane: functions and related enzymes. Biol Pharm Bull. 2006;29:1542.

Ready to submit your research? Choose BMC and benefit from

- fast, convenient online submission

- thorough peer review by experienced researchers in your field

- rapid publication on acceptance

- support for research data, including large and complex data types

- gold Open Access which fosters wider collaboration and increased citations

- maximum visibility for your research: over $100 \mathrm{M}$ website views per year

At BMC, research is always in progress.

Learn more biomedcentral.com/submission 